\title{
EXPERIMENTAL INVESTIGATION INTO HYDROGEN PRODUCTION THROUGH ALKALINE WATER ELECTROLYSIS
}

\author{
I.M. Sakr, A-F.M. Mahrous, A. Balabel, and K. Ibrahim \\ Mechanical Power Engineering Dept., Faculty of Engineering \\ Minoufiya University, Shebin El-Kom, Egypt \\ Email: ismailsakr@yahoo.com,afmahrous@hotmail.co.uk,ashrafbalabel@yahoo.com
}

\begin{abstract}
Alkaline water electrolysis is considered to be a basic technique for hydrogen production. Many researchers have investigated the water electrolysis in order to promote electrochemical reaction. The effects of voltage, solution concentration, and space between the pair of electrodes on the amount of hydrogen produced and consequently on the overall electrolysis efficiency are experimentally investigated. The alkaline water electrolysis of different potassium hydroxide aqueous solutions is conducted under atmospheric pressure using stainless steel electrodes. The experimental results showed that, the performance of alkaline water electrolysis unit is highly affected by the voltage input and the gap between the electrodes. Higher rates of produced hydrogen can be obtained at smaller space between the electrodes and also at higher voltage input. Higher system efficiency was also gained at smaller gap distances between the pair of electrodes.

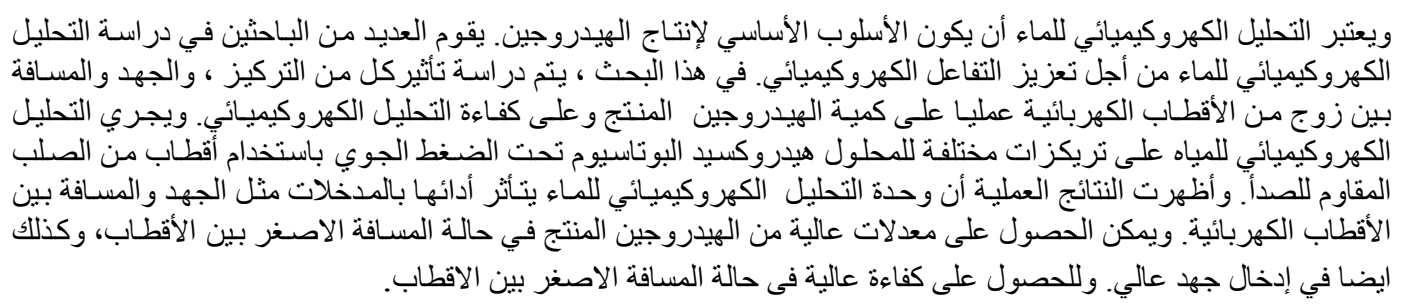

Keywords: Hydrogen production, alkaline water electrolysis, experimental measurements.

\section{INTRODUCTION}

Hydrogen plays an important role as an energy carrier for sustainable development [1-5]. It can be used in fuel cells to generate electricity, with water as the only product [6]. Besides, hydrogen is considered as the lightest element and any leakage of hydrogen gas can disperse quickly and thus hydrogen is believed as safe as other commonly used fuels [7]. Hydrogen could be produced from other sources with energy input. Presently, hydrogen can be economically produced from hydrocarbon reforming, which is neither renewable nor clean from the life cycle point of view [6-8]. Powered by solar energy, hydrogen can be produced from water via photocatalysis, thermochemical cycles, and water electrolysis [7-10]. These methods offer renewable and clean production of hydrogen fuel and, therefore, have attracted increasing research interests in recent years. So far, the efficiencies of photocatalysis and thermochemical cycles are still too low to be economically competitive. Water electrolysis is hence the most promising technology for large-scale hydrogen production [5,9]. Compared with traditional alkaline electrolysis, in which potassium hydroxide $(\mathrm{KOH})$ solution is used as the electrolyte, the advantages of the alkaline electrolyzer include simplicity, proven technology, and no special requirements, Disadvantages of the alkaline electrolyzer are the low efficiency and low current density [11-12].

In the literature, the hydrogen molecules accumulate on the surface of the electrode until a bubble forms, breaks away, and rises to the surface of the electrolyte. At the oxygen electrode, a similar process occurs in which hydroxyl ions are discharged by giving up their electrons to the electrode and reacting to form water and oxygen. The oxygen molecules accumulate into gas bubbles and rise to the surface. Le Roy et al. [13] pointed out that, the increase of volume fraction of hydrogen or oxygen bubbles between the electrodes would cause an increase in the electric resistance in aqueous solution, resulting in efficiency decrease of water electrolysis. Most of previous research studied the effect of current, distance between the electrodes, and the temperature on efficiency of water electrolysis at a particular concentration of the solution [14-15]. In the present work, stainless steel electrodes have been 
used for hydrogen production from selected various concentration of electrolyte solutions at various input power and with a variable distance between the electrodes to study its effect on electrolysis efficiency.

\section{EXPERIMENTAL APPROACH}

In the following section, the design of the test models for the electrochemical water analysis device is illustrated. An approach to improve the performance of the electrolysis system through a number of experimental runs is also suggested.

In order to investigate the parameters affecting hydrogen production through water electrolysis, a set of experimental test models were designed and manufactured. Figure 1 shows the experimental apparatus for alkaline water electrolysis. The water electrolysis of potassium hydroxide $(\mathrm{KOH})$ aqueous solution was conducted under atmospheric pressure. The experimental test model consists of the following components: a DC power supply, a transformer (one fixed- one variance), a rectifier, a capacitor, electrodes, an electric wire, a thermocouple, and an avometer.

An AC powered unregulated power supply usually uses a transformer to convert the voltage from the mains to a different, usually a lower, voltage. If it is used to produce DC, a rectifier is used. A capacitor is further used to smooth the pulsating current from the rectifier. Some small periodic deviations from smooth direct current may remain, which is known as ripple. These pulsations occur at a frequency related to the AC power frequency. The simplest unregulated DC power supply circuit consists of a single diode and resistor in series with the AC supply. This circuit is common in rechargeable flashlights.
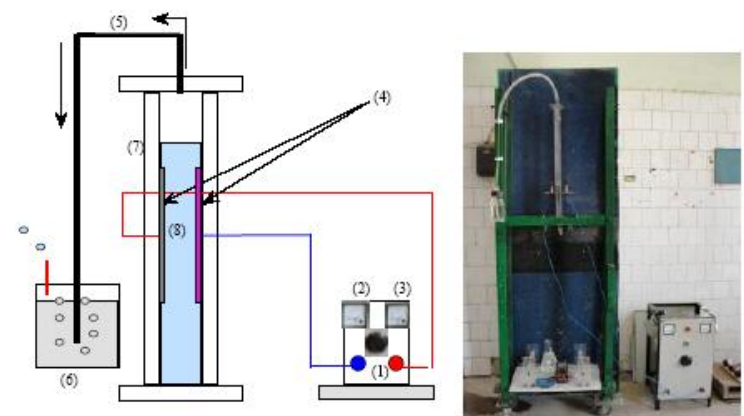

1. DC Power supply,

2.Voltmeter, 3.Ammeter,

4.Electrodes,

7.Elecrolyser,

5.PVC Pipe, 6.Bubbler,

8. Aqueous solution.

Fig. 1 Experimental apparatus for the alkaline water electrolysis

An electrode is a member in an electrochemical cell. Electrode is referred to as either an anode or a cathode. The anode is now defined as the electrode at which electrons leave the cell and oxidation occurs, and the cathode as the electrode at which electrons enter the cell and reduction occurs. Each electrode may become either the anode or the cathode depending on the voltage applied to the cell. A bipolar electrode is an electrode that functions as the anode of one cell and the cathode of another cell. The specifications of electrodes were: number of plates is two (one anode-one Cathode), material is 316LHS Stainless steel, area dimension is $5 \mathrm{~cm} \times 10 \mathrm{~cm}$, and plate thickness is $3 \mathrm{~mm}$. A $6 \mathrm{~mm}$ thickness of electric wire was used to transfer the current from the DC power supply to the electrodes.

\section{TEST MODELS}

The test models were manufactured from Plexiglas with plate height of $30 \mathrm{~cm}$, width of $6 \mathrm{~cm}$, and thickness of $0.5 \mathrm{~cm}$. Four test models were produced depending on the distance between the electrodes. Referring to the gap between the electrodes as "Delta $(\delta)$ ", it takes the values of $3 \mathrm{~mm}$ for the first model (M1), $5 \mathrm{~mm}$ for the second model (M2), $8 \mathrm{~mm}$ for the third model (M3), and $20 \mathrm{~mm}$ for the fourth model (M4). Figure 2 shows the experimental test model for M1.

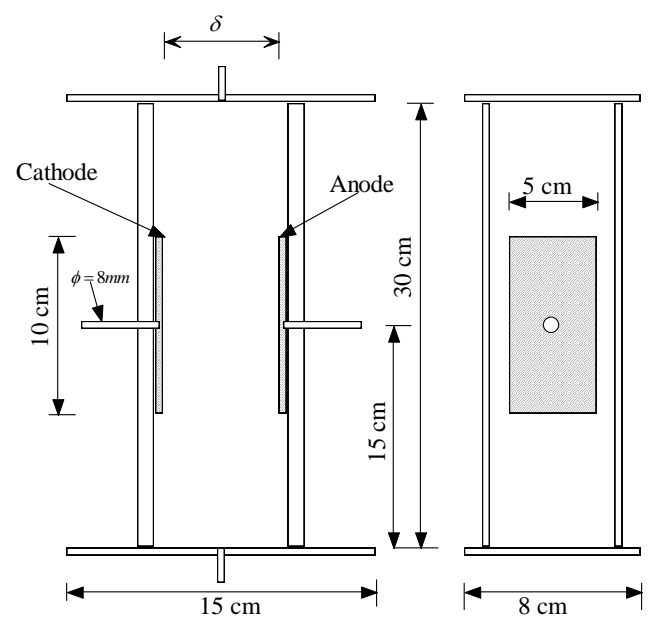

Fig. 2 Elevation (left) and side (right) views of the experimental test model

Before starting the test, all components must be in their right position. The solution should then be prepared to give the desired $\mathrm{KOH}$ concentration in pure water. The electrodes are positioned in the electrolysis vessel and the water electrolysis is started to function at certain electric voltage. The cell voltage, current, and water mass before and after the test are measured within a specific period of time. The voltage is then changed to run the cell under the same condition of $\mathrm{KOH}$ concentration. Uncertainty in the measurements are $\pm 1 \%$ for mass, $\pm 2 \%$ for current (I) and $\pm 0.35 \%$ for volt. 


\section{RESULTS AND DISCUSSION}

This section explores the influence of gap between the two electrodes and the concentration of potassium hydroxide on the hydrogen generation process. To fulfill a comprehensive understanding of the effects of the space between the two electrodes on the rate of hydrogen production, four different gaps were examined. During each test, the pair of electrodes were exposed to a DC volt ranges from 2 to 5 volts. Different concentrations of electrolyte solution were also tested. The percentage water mass lost during a specific time was measured and the corresponding system efficiency was calculated using the mass ratio of the hydrogen in a single molar mass of water.

\subsection{Effect of Gap between the Two Electrodes}

The rate of hydrogen production, expressed in terms of percentage loss in water cell mass, as a function of the input voltage is shown in Fig. 3 for different gaps between the electrodes and at $10 \%$ of the potassium hydroxide concentration (i.e. solution strength as \% of solute in the solution by mass). Figure 3 shows that, the percentage loss in water mass is greatly affected by the degree of voltage strength at the terminals of the electrodes. At a constant space between the two electrodes, when the voltage increases the amount of lost mass increase. The resulting increase in the percentage mass lost due to the increase in the voltage is attributed to the increase in electrical current.

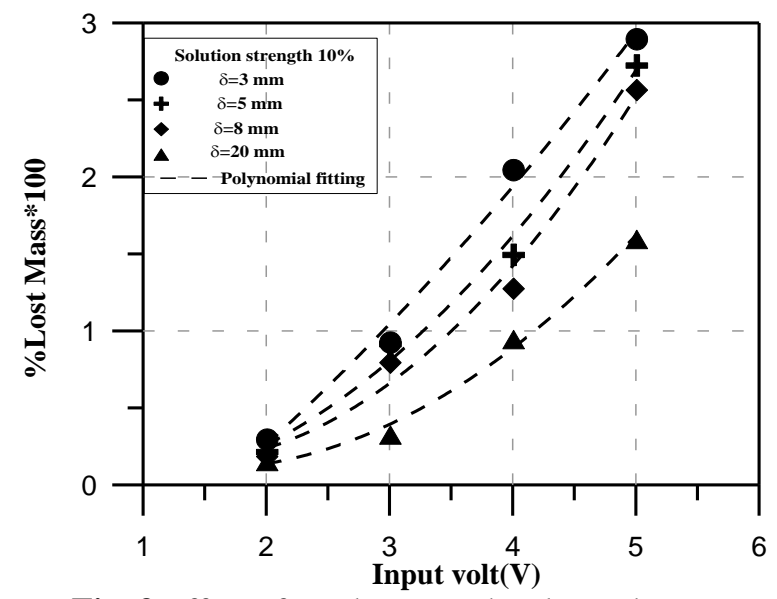

Fig. 3 Effect of gap between the electrodes on hydrogen production rate

The results displayed in Fig. 3 show also that the percentage mass lost depends to on the space between the two electrodes. When the space between the pair of electrodes decreases the percentage lost of water mass increases due to the resulting decrease in electrical resistance between the electrodes that in turn leads to an increase in the electrical current [14]. The increase in the hydrogen production rate due to the decrease in the space between the electrodes becomes obvious at higher degrees of input voltage.
The efficiency of the water electrolysis system as a measure of its hydrogen production capacity is displayed in Fig. 4. Mathematically it is the ratio between the rate of combustion energy of the produced hydrogen and the electric power input to the system. The overall system efficiency can be calculated as follows:

Efficiency = Output Power/ Input Power

Input Power $=$ Voltage $\mathrm{x}$ Current

Output Power $=$ Combustion Energy $/$ Time (seconds)

$$
2 \mathrm{H}_{2}+\mathrm{O}_{2} \leftrightarrow 2 \mathrm{H}_{2} \mathrm{O} \quad(\text { Heat }=-572 \mathrm{~kJ})
$$

Combustion Energy $=$ moles $\mathrm{H}_{2} \times(-572 \mathrm{~kJ})$

$$
=----\mathrm{kJ} \text { released }
$$

Moles $\mathrm{H}_{2}=$ Lost mass $\left(2 \mathrm{H}_{2}+\mathrm{O}_{2}\right) \times 1 \mathrm{~mol}\left(2 \mathrm{H}_{2}+\mathrm{O}_{2}\right) \times$ $2 \mathrm{~mol} \mathrm{H}_{2} /\left(39.09 \times 1\right.$ moles $\left(2 \mathrm{H}_{2}+\mathrm{O}_{2}\right)$

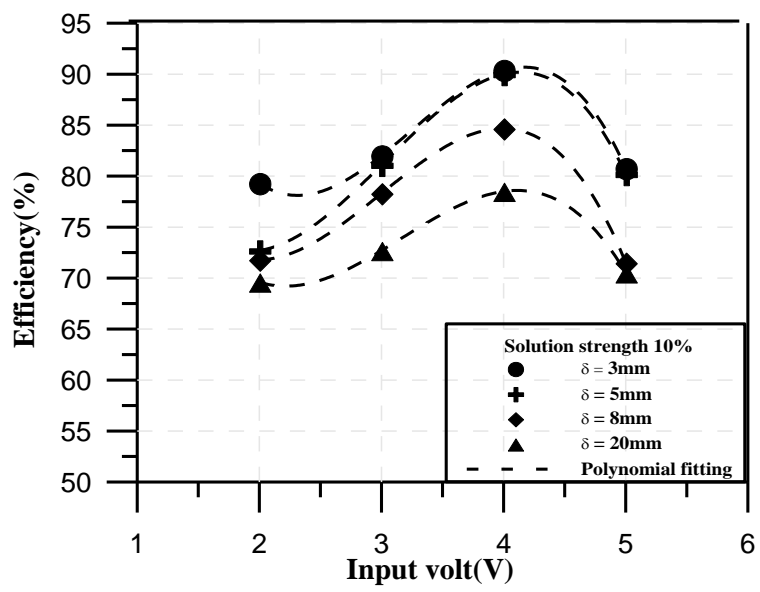

Fig. 4 Effect of gap between the electrodes on water electrolysis efficiency

As shown in Fig. 4, higher system efficiency can be obtained at small gaps between the pair of electrodes. An important as well as interesting result can be observed from Fig. 4 concerning the input voltage corresponding to the maximum efficiency. By increasing the input voltage, for each distance, the efficiency increases up to a specified voltage, shown in Fig. 4 (4 volt), then the efficiency decreases by increasing the input voltage. This can be explained by the increase of bubble production and the accumulation on the electrodes surfaces causing a resistance to the process and a reduction of the overall system efficiency.

\subsection{Effect of Solution Strength on the Water Mass Lost}

The effects of potassium hydroxide concentration of the electrolyte solution on the system performance are depicted on Figs. 5 and 6. Figure 5 demonstrates that, as the concentration of the potassium hydroxide in the solution increase, the rate of hydrogen production also increases. 


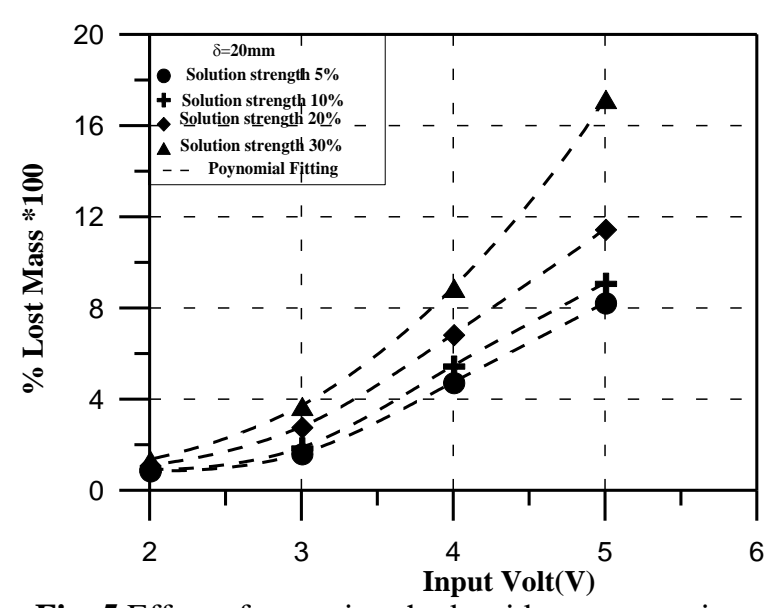

Fig. 5 Effect of potassium hydroxide concentration on the rate of water mass lost

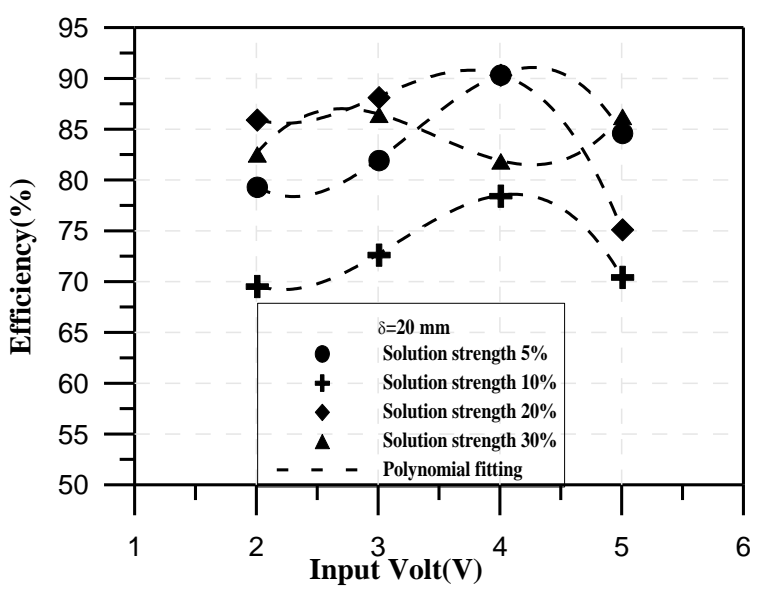

Fig. 6 Effect of potassium hydroxide concentration on water electrolysis efficiency

This behavior is attributed to the increase in the electrical conductivity of the solution due to the increase in the concentration of the potassium hydroxide. Increasing the solution electrical conductivity leads to an increase in the electrical current passing through the solution and consequently to an increase in water lost mass. The measured data on Fig. 6 shows that, the efficiency does not have a common trend with the voltage at different degrees of solution concentrations. When the voltage has strength between 3.5 and 5 volts, the system efficiency reaches its maximum value. Consequently, an extended study is required in more details. This can be considered in a future study.

Experimental data is used to deduce correlation equation (3). Figure 7 shows the relation between maximum efficiency and gap between the electrodes on water electrolysis.

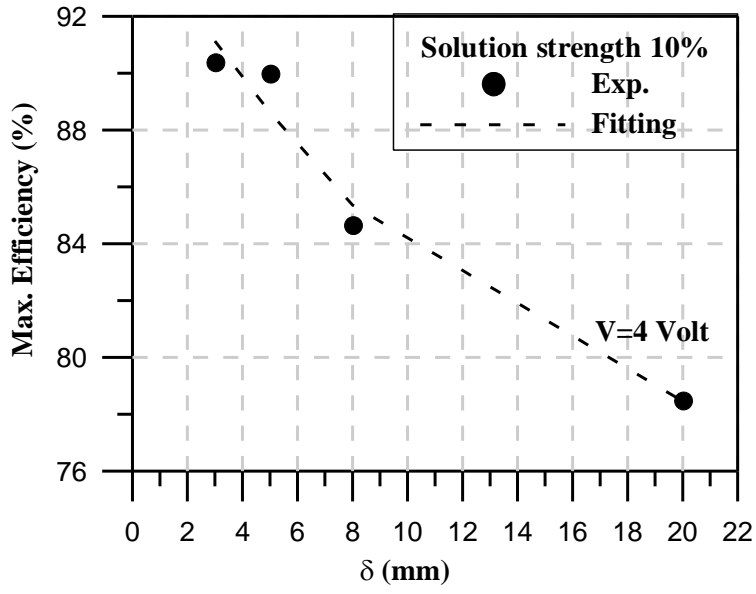

Fig. 7 Effect of gap between the electrodes on maximum water electrolysis efficiency

$$
\eta_{\max }=95.411-1.528 \delta+.034 \delta^{2}
$$

\section{CONCLUSION}

The current research is aimed to conduct an experimental investigation of alkaline water electrolysis for the purpose of hydrogen production. The investigation covered the effects of voltage, solution concentration, and space between the pair of electrodes on the characteristics of alkaline water electrolysis. The study was carried out under atmospheric pressure using stainless steel electrodes. The experimental results showed that, the performance of water electrolysis unit is dominated by operational parameters like the voltage input and the gap between the electrodes. Smaller gaps between the pair of electrodes and higher input voltage were demonstrated to produce higher rates of hydrogen at higher system efficiency. The overall system maximum efficiency increases to a specified input voltage and then it decreases by further increasing of the input voltage. A correlation for the maximum water electrolysis efficiency has been developed.

\section{REFERENCES}

[1] L. D.Silva, A. Bergel, D. Feron, and R. Basseguy, "Hydrogen production by electrolysis of a phosphate solution on a stainless steel Cathode", International Journal of Hydrogen Energy, 35 (2010), pp: 8561-8568.

[2] K. Zeng, and D. Zhang, "Recent progress in alkaline water electrolysis for Hydrogen production and applications", Progress in Energy and Combustion Science, 36(2010), pp: 307-326.

[3] G. Wei, Y. Wang, C. Huang, Q.G.Zhitao, and L. $\mathrm{Xu}$," The Stability of MEA in SPE water electrolysis for hydrogen production", International Journal of Hydrogen Energy 35 (2010), pp: 3951-3957. 
[4] M. Ni, M. K.H. Leung, K. Sumathy, and D.Y.C. Leung," Potential of Renewable hydrogen production for energy supply in Hong Kong", International Journal of Hydrogen Energy 31(2006), pp: 1401-1412.

[5] A.Balabel, I.M. Sakr, and K. Ibrahim, "Potential of Renewable Hydrogen Production Technologies For energy requirements in Egypt", Seventh Conference Of Egyptian Rural Development Water, energy and rural development, Faculty of Engineering, Menoufia University, 13-15 October 2009.

[6] I. Schulte, D. Hart, and R. van der Vorst," Issues affecting the acceptance of Hydrogen fuel", International Journal of Hydrogen Energy 29(2004), pp: 677-685.

[7] N. Lymberopoulos, "Hydrogen Production from Renewable", Centre for Renewable Energy Sources; 2005.

[8] C. Koroneos, A. Dompros, G. Roumbas, and N. Moussiopoulos, "Life cycle assessment of Hydrogen fuel production processes", International Journal of Hydrogen Energy, 29(2004), pp: 1443-1450.

[9] J. Nie, Y. Chen, R. F. Boehm, and S. Katukota, "A Photoelectrochemical Model of Proton Exchange Water Electrolysis for Hydrogen Production”, J. Heat Transfer 130, (2008).
[10] W.G. Colella, M.Z. Jcobson, and D.M. Golden, "Switching to a U.S. hydrogen fuel cell Vehicle fleet: the resultant change in emissions, energy use, and greenhouse gases", Journal of Power Sources, 150 (2005), pp: 150-181.

[11] O. Ulleberge, "Modeling of advanced alkaline electrolyzers: a system approach"; J of Hydrogen energy, 28(2003), pp: 21-23, 2003.

[12] S. Srinivasan, and F.J. Salzano, "Prospects for hydrogen production by water electrolysis to be competitive with conventional methods"; Int J Hydrogen Energy; 2(1977), pp: 53-59.

[13] H. Riegel, J. Mitrovic and K.Stephan,"Role of Mass Transfers on Hydrogen evolution in Aqueous Media", J. Appl.Electrochem 28(1998), pp: 10-17.

[14] N. Nagai, M.Takeuchi, T. Kimura, and T.Oka, "Existence of optimum space between Electrodes on hydrogen production by water Electrolysis"; Int J Hydrogen Energy, 28(2003), pp: $35-41$.

[15] N. Nagai, M. Takeuchi, and M,T. Furuta, "Effects of bubbles between electrodes $n$ alkaline Water Electrolysis Efficiency under forced convection of electrolyte"; WHEC 16 / 13-16 June 2006, Lyon France, pp: 1-10. 